\title{
Benefícios Sociais da Política de Crédito Rural Destinada à A vicultura de Corte no Brasil
}

\author{
Valéria Avinte de Almeida e N eves* \\ Ahmad Saeed Khan** \\ Patricia V.P. Sales Lima***
}

Resumo: Neste artigo, procurou-se avaliar o impacto social do crédito rural destinado à avicultura de corte brasileira. 0 modelo utilizado foi o de Lindner e J arret, modificado por Rose, considerando deslocamentos paralelo e pivotal da oferta. O s resultados mostraram que a política de crédito rural destinada à avicultura brasileira gerou benefícios sociais, considerando deslocamento paralelo da curva. Quando considerado custo médio constante, deslocamento pivotal da oferta, o benefício total gerado resulta em $\mathrm{R} \$ 102.135 .880,94$, o que revela a importância de ações voltadas para a redução nos custos de produção como forma de estimular o desenvolvimento da avicultura no país.

Palavras-chave: Avicultura, Benefício Social, Crédito Rural.

Abstract: This study tried to evaluate the social impact of agricultural credit destinated to brazilian poultry meat production. The model developed by Linder and J arret modified by Rose wasused. The parallel and pivotal shift in supply curve was considered. The results suggested that agricultural credit policy to increase poultry meat production generated social benefits. Howere, the benefits produced by parallel shift in supply curve were greater than those created by pivotal shift. Considering the average cost constant, the social benefits of worth $R \$ 102,135,880.94$ were produced. The results showed the importance of agricultural credit policy of reducing cost of production and stimulating the development of poultry production in the country.

Keyw ords: Poultry Meat Production, Social Benefits, Agricultural Credit.

J EL Classification: Q130; Q180

\section{Introdução}

O desenvolvimento da atividade avícola no Brasil se deu a partir de 1960, quando tiveram início as importações de linhagens híbridas

\footnotetext{
Economista, Mestre em Economia Rural pela Universidade Federal do Ceará. E-mail: valeria20neves@hotmail.com.

* Professor Titular do Departamento de Economia Agrícola da Universidade Federal do Ceará. Bolsista de Produtividade CNPq. E-mail: saeed@ufc.br.

*** Professora Adjunto do Departamento de Economia Agrícola da Universidade Federal do Ceará. Bolsista de Produtividade CNPq. E-mail: pvpslima@gmail.com.
} 
americanas de frango. Somado a isso, ocorreu a implementação de novas tecnologias no campo da genética, da nutrição e da sanidade animal. Como resultado, em 2005, o Brasil foi o segundo maior produtor mundial de frango de corte. (ABEF, 2006.)

A atividade está presente em todas as regiões do país, impactando a economia da maioria dos estados. No Sul do país, encontra-se $59,9 \%$ da produção nacional, a região Sudeste participa com $25,1 \%$ e 0 Centro-O este com $11,2 \%$. No ano de 2005 , o Paraná participou com 22,83\% do abate de frangos com SIF (Sistema de Inspeção Federal), vindo após Santa Catarina com 16,76\%, Rio Grande do Sul com 14,76\% e São Paulo com 14,43\%. (ABEF, 2005)

O setor é um grande gerador de renda e responsável, aproximadamente, por 4 milhões de empregos diretos e indiretos, conforme a AVIMIG - Associação dos Avicultores de Minas Gerais (2007). Atualmente, a avicultura representa 1,5\% do PIB, gerando R\$ 6 biIhões apenas em impostos. Do total da produção avícola, $70 \%$ são destinados ao mercado doméstico, com consumo de $35 \mathrm{~kg}$ de carne e 128 ovos per capita. (AVIMIG, 2007.) Diante dessa perspectiva vislumbra-se a importância econômica e social da atividade avícola no contexto da agropecuária nacional.

No intuito de promover maior desenvolvimento da agropecuária nacional, o governo vem adotando estratégias que fomentam a atividade avícola. Dentre os instrumentos de política agrícola, encontra-se o crédito rural. A oferta de crédito rural destinado à agropecuária nacional, em 1997, aumentou mais de 100\%, em comparação a 1995, segundo o Ministério do Planejamento e Orçamento (1998). Houve um maior direcionamento do Governo às aplicações obrigatórias dos bancos, que cresceram tanto em termos relativos quanto absolutos, pois ocorreu um aumento dos depósitos à vista, devido à queda da inflação e da elevação dos percentuais das exigibilidades bancárias.

A ampliação do crédito rural para a agropecuária impactou diretamente a expansão do crédito rural destinado à avicultura. Pois, observou-se um acréscimo de 138 e 151\% na oferta de crédito rural para o setor avícola nos anos de 1997 e 2004, respectivamente, conforme dados obtidos junto ao A nuário de Avicultura Industrial (2004).

A política de crédito rural consiste no financiamento à agropecuária e teve início em 1930, através da criação da Carteira de Crédito Agrícola e Industrial - CREAI -, pelo Banco do Brasil, cujo objetivo era suprir a inexistência de um sistema financeiro capaz de gerar recursos por meio de emissão de títulos (SALES, 2005).

Atualmente, a política agrícola nacional é regida pela Lei Federal $n$ ㅇ 8.171, de 17 de janeiro de 1991, que dispõe sobre a política agrícola e, especificamente no capítulo XIII, dispõe sobre o crédito rural. Segundo esta Lei, o crédito rural tem como principais objetivos: 
I) estimular os investimentos rurais para produção, extrativismo não predatório, armazenamento, beneficiamento e instalação de agroindústria, sendo esta quando realizada por produtor rural ou suas formas associativas;

II) favorecer o custeio oportuno e adequado da produção, do extrativismo não predatório e da comercialização de produtos agropecuários;

III) incentivar a introdução de métodos racionais no sistema de produção, visando ao aumento da produtividade, à melhoria do padrão de vida das populações rurais e à adequada conservação do solo e preservação do meio ambiente;

IV) propiciar, através de modalidade de crédito fundiário, a aquisição e a regularização de terras pelos pequenos produtores, pelos posseiros e pelos arrendatários e trabalhadores rurais.

A ampliação do crédito rural para a agropecuária impactou diretamente a expansão do crédito rural destinado à avicultura. Pois, observou-se um acréscimo de 138 e $151 \%$ na oferta de crédito rural para o setor avícola nos anos de 1997 e 2004, respectivamente, conforme dados obtidos junto ao A nuário de Avicultura Industrial (2004).

Dado que uma política de crédito rural deve fortalecer alianças mercadológicas e cadeias produtivas além de estimular o bemestar da sociedade desde o meio rural até à zona urbana, é de se esperar que o crédito rural destinado à avicultura tenha provocado benefícios sociais. Neste sentido, a proposta do presente estudo é realizar uma análise dos benefícios sociais da política de crédito rural destinada à avicultura de corte brasileira.

\section{Referencial Teórico}

\subsection{Excedente econômico do consumidor e do produtor}

0 excedente do consumidor é a diferença entre o que o consumidor está disposto a pagar por determinado bem e o que efetivamente paga. 0 excedente do consumidor tem aplicações importantes em economia, quando é somado o de muitas pessoas. Neste caso, ele mede o benefício agregado que os consumidores obtêm quando adquirem produtos em um mercado. $O$ excedente do consumidor pode ser medido pela área abaixo da curva de demanda e acima da linha que representa o preço efetivo de aquisição do bem.

O excedente do produtor é a diferença entre a quantia mínima pela qual o produtor está disposto a vender determinada mercadoria e a quantia pela qual realmente vende esta determinada mercadoria. (VARIAN, 2003.) 
Os instrumentos de excedente econômico do consumidor e do produtor podem ser usados para calcular os benefícios e custos de várias políticas econômicas.

\subsection{Benefício social total}

A análise do benefício social é um instrumental frequentemente utilizado para avaliação dos resultados obtidos com a implementação de determinados projetos, com vistas a verificar seus impactos sociais. Assim, pode-se perceber se a sociedade como um todo ficará em melhor situação empreendendo, ou não, determinado projeto, ou então empreendendo uma série de projetos alternativos.

Basicamente dois modelos destacam-se na análise dos benefícios sociais de políticas agrícolas. Primeiramente, o modelo de análise da função de produção que envolve a estimativa da produtividade marginal de uma política agrícola. Contudo, tal modelo apresenta algumas desvantagens. Por ser um modelo empregado em análises ex post, sua aplicação requer um número considerável de observações passadas, tornando-se inadequado para pesquisas que envolvam apenas dados recentes. (CRUZ et al., 1982.)

Segundo, o modelo conhecido como método de número-índice ou método de custo-benefício direto, que mede a produtividade média. Este método possui grande relevância dentro da literatura de cálculo do benefício social, pois permite ser utilizado tanto em análise ex-post (considerando os benefícios sociais que as políticas empregadas trouxeram) como em análises "ex-ante" (considerando os benefícios sociais que as políticas empregadas poderão trazer), bastando, para isso, estimar o deslocamento da curva de oferta resultante das políticas agrícolas implementadas para um dado produto. (CRUZ et al., 1985).

Este modelo baseia-se no conceito de "excedente econômico" de Marshall e, segundo Khan e Souza (1991), as suas principais premissas são:

I) A área total sob a curva de demanda à esquerda de uma dada quantidade representa a utilidade total desta quantidade;

II) A curva de oferta reflete os custos de oportunidade dos recursos variáveis utilizados para produzir cada quantidade.

Com relação à teoria do excedente econômico de Marshall, muitos trabalhos foram realizados em diversos lugares do mundo. Alguns deles são citados a seguir:

Griliches (1958) estimou os benefícios para a sociedade com a descoberta do milho híbrido, considerando oferta e demanda lineares 
e deslocamento paralelo para a direita. No entanto, ele supôs dois casos: primeiro, para curvas de oferta perfeitamente elásticas e, segundo, para curvas de ofertas perfeitamente inelásticas.

Peterson (1967) analisou o benefício social bruto da pesquisa em aves, considerando as curvas de oferta e demanda de especificação generalizada, com deslocamento proporcional da curva de oferta.

Schmitz e Seckler (1970) avaliaram os ganhos sociais da agricultura mecanizada, para o caso da colhedeira de tomate. 0 modelo utilizado foi de curvas de oferta e demanda lineares. Os autores consideraram que a oferta não é perfeitamente elástica e seu deslocamento paralelo.

Ayer e Schuh (1972) estimaram os impactos econômicos dos investimentos empregados na pesquisa de algodão no Estado de São Paulo. Consideraram curvas de oferta e demanda não-lineares, com deslocamento pivotal. Os autores estimaram taxa de retorno variando entre $77 \%$ e $110 \%$ ao ano.

A kino e Hayami (1975) usaram um modelo semelhante ao anterior, para estimar os benefícios da pesquisa em melhoramento do arroz no J apão. Admitiram curvas de oferta e demanda com elasticidades-preço unitária, com deslocamento pivotal. Incluíram na análise os efeitos distributivos da política de importação do arroz.

Hertford e Schmitz (1977) avaliaram um modelo semelhante ao de Peterson, porém com curvas de demanda e oferta lineares, considerando um deslocamento paralelo.

Hayami e Herdt (1977) estimaram os benefícios sociais do arroz nas Filipinas, supondo curvas de oferta e demanda com elasticidades-preço constantes e deslocamento pivotal da curva de oferta. Incluíram o autoconsumo, considerando o produto como parte da subsistência.

Apesar da contribuição inconteste que os estudos citados proporcionaram, Lindner e J arret (1978) argumentaram que todas as técnicas anteriores utilizadas para avaliar os benefícios sociais brutos da pesquisa podem levar a resultados tendenciosos, exatamente porque pouca atenção tem sido dada ao modelo de variação na curva de oferta em resposta à adoção de novas tecnologias pelos produtores rurais. Os autores consideraram curvas de oferta e demanda lineares e demonstraram que os benefícios sociais anuais decorrentes da adoção de uma nova tecnologia pelos produtores rurais são influenciados pela natureza do deslocamento da curva de oferta e analisaram quatro situações de deslocamento: convergente, paralelo, divergente pivotal e divergente proporcional. 
Segundo Rose (1980), os benefícios brutos da pesquisa encontrados por Lindner e J arret tiveram seus valores subestimados e sugeriu equações alternativas para obtenção dos preços e quantidades futuras.

Santana e Khan (1987), estimando os retornos sociais gerados com a adoção tecnológica na cultura do feijão cauípi no Nordeste, utilizaram o modelo proposto por Lindner e J arret, considerando curvas de oferta e demanda lineares e deslocamento divergente-proporcional da curva de oferta. Os autores estimaram uma redução de aproximadamente $10 \%$ no custo de produção de feijão e os produtores e os consumidores receberam sessenta e nove milhões de cruzados (moeda vigente no ano da pesquisa) em benefícios sociais.

Khan e Souza (1991), avaliando os impactos socioeconômicos dos investimentos em pesquisa na cultura de mandioca no Nordeste, também utilizaram o modelo de Lindner e J arret, porém levando em conta a modificação sugerida por Rose, considerando curvas de oferta e demanda lineares, deslocamento divergente proporcional da curva de oferta e o autoconsumo. Os benefícios sociais foram maiores quando o deslocamento considerado da curva de oferta foi divergente proporcional.

Silva e Khan (1994) calcularam os benefícios sociais da substituição de milho por raspa de mandioca no uso da ração suína, no Estado do Ceará. Neste trabalho, também foi utilizado o modelo modificado por Rose (1980), considerando curvas de oferta e demanda lineares e os deslocamentos paralelo, divergente-proporcional e convergente da curva de oferta.

Abdallah (1998) calculou os benefícios sociais da política de incentivos fiscais à pesca no Brasil, utilizando também o modelo de Rose (1980), considerando curvas de demanda e oferta lineares e deslocamentos pivotal e paralelo da curva de oferta

A abordagem da análise do custo-benefício direto será a utilizada no presente estudo. Especificamente, será utilizado o modelo desenvolvido por Lindner e J arret (1978), aperfeiçoado por Rose (1980), considerando curvas de demanda e de oferta lineares e os deslocamentos pivotal e paralelo da curva de oferta .

$O$ acréscimo no excedente econômico advindo do deslocamento paralelo da curva de oferta é representado pela área $A_{0} M_{0} M_{1} A_{1} A_{0}$ $(Y+X)$ (Figura 1$)$. 


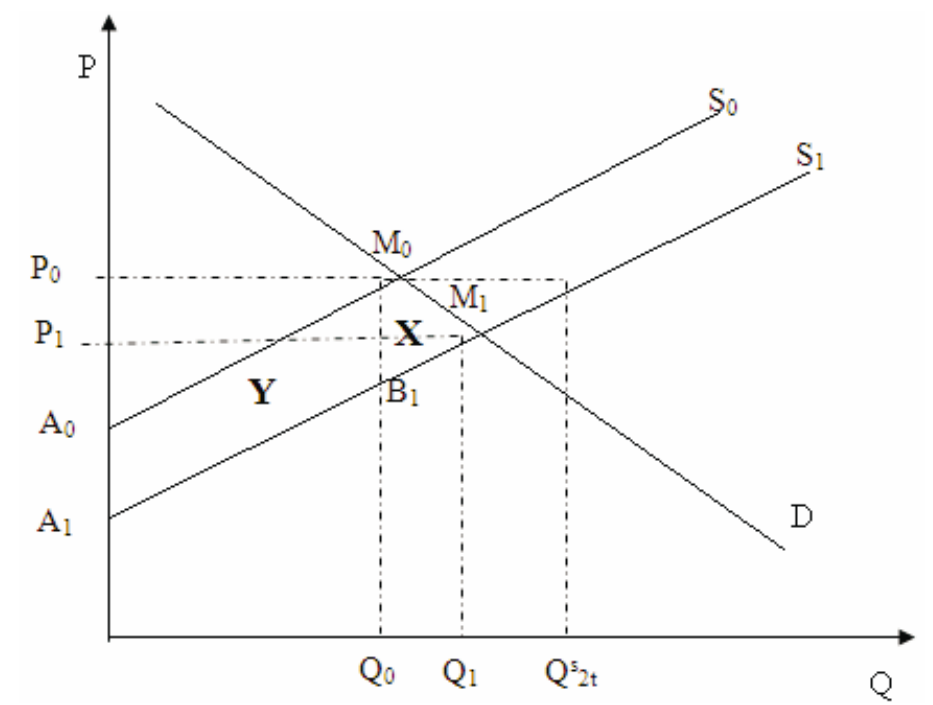

Figura 1 - Benefício social total advindo de um deslocamento paralelo da curva de oferta agregada.

Segundo Rose (1980), para uma mudança paralela na curva de oferta, a área que representa o aumento do excedente econômico, ou benefício social total, pode ser estimada, separadamente, somando $M_{0} M_{1} B_{1} M_{0}(X)$ com $A_{0} M_{0} B_{1} A_{1} A_{0}(Y)$, especificando de forma clara 0 valor de $M_{0} B_{1}$, que representa a mudança no custo unitário devido ao deslocamento da curva de oferta. Assim, o benefício total é dado pela equação abaixo:

$$
B S T=X+Y
$$

A operacionalização das áreas $X$ e $Y$ é apresentada a seguir. Segundo Abdallah (1998), no ponto de coordenadas $\left(Q_{2}^{s} t, P_{0}\right)$, na Figura 1, tem-se que a elasticidade-preço da oferta $\left(\varepsilon^{s}\right)$ é:

$$
\left(\varepsilon^{S}\right)=\frac{\left(\frac{Q_{2 t}^{s}-Q_{0}}{Q_{2}^{s} t}\right)}{\frac{M_{0} B_{1}}{P_{0}}}
$$


A eficiência da implantação da política se traduz na redução proporcional do custo unitário, que será denominado de $K$, provocando um deslocamento da curva de oferta. Esta mudança no custo unitário pode ser calculada da seguinte forma:

$$
K=\frac{M_{0} B_{1}}{P_{0}}
$$

Substituindo a expressão (3) em (2), tem-se:

$$
\left(\varepsilon^{S}\right)=\frac{\left(1-\frac{Q_{o}}{Q_{2}^{S} t}\right)}{K} \quad \therefore \quad K=\frac{\left(1-\frac{Q_{o}}{Q_{2}^{S} t}\right)}{\left(\varepsilon^{S}\right)}
$$

Da expressão (3), tem-se:

$$
K . P_{0}=M_{0} B_{1}
$$

Com esses dados, pode-se agora determinar a expressão algébrica das áreas $\mathrm{X}$ e $\mathrm{Y}$, chegando, assim, à expressão do aumento do Benefício Social Total (BST).

$A$ área $Y\left(A_{0} M_{0} B_{1} A_{1} A_{0}\right)$ pode ser derivada da seguinte forma: Observando-se a Figura 2, toma-se a área do retângulo $\left(R=X_{1}+Y\right.$ $\left.+X_{2}\right)=A_{1} P_{0} M_{0} N A_{1}$ e subtrai-se destas as duas áreas dos triângulos $\left(X_{1}\right.$ e $\left.X_{2}\right)$, respectivamente, $X_{1}=A_{0} P_{0} M_{0} A_{0}$ e $X_{2}=A_{1} B_{1} N A_{1}$.

Sendo:

Área do retângulo $R=Q_{0^{*}}\left(P_{0}-A_{1}\right)$;

Área do triângulo $\mathrm{X}_{1}=0,5 \mathrm{Q}_{0^{\circ}}\left(\mathrm{P}_{0}-\mathrm{A}_{0}\right)$;

Área do triângulo $X_{2}=0,5 Q_{0} .\left(B_{1}-A_{1}\right)$; 


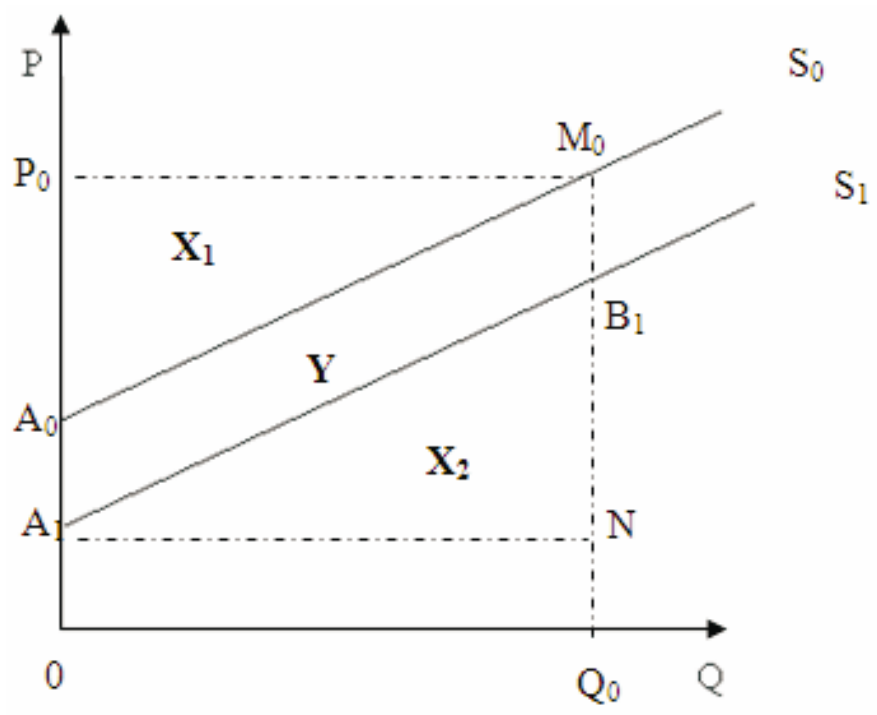

Figura 2 - Demonstração geométrica do cálculo da área Y

Tem-se :

$$
Y=Q_{0}\left(P_{0}-A_{1}\right)-0,5 Q_{0}\left(P_{0}-A_{0}\right)-0,5 Q_{0}\left(B_{1}-A_{1}\right)
$$

Ainda de acordo com Abdallah (1998), define-se o segmento $M_{0} B_{1}$ como sendo $C=P_{0}-B_{1}$. Fazendo-se $B_{1}=P_{0}-C$, substitui-se $a$ última expressão na equação acima, obtendo-se:

$$
\begin{aligned}
& Y=Q_{0}\left(P_{0}-A_{1}\right)-0,5 Q_{0}\left(P_{0}-A_{0}\right)-0,5 Q_{0}\left(P_{0}-C-A_{1}\right) \\
& Y=0,5 Q_{0}\left[2\left(P_{0}-A_{1}\right)-P_{0}+A_{0}-P_{0}+C+A_{1}\right] \\
& Y=0,5 Q_{0}\left(2 P_{0}-2 A_{1}-2 P_{0}+A_{0}+C+A_{1}\right) \\
& Y=0,5 Q_{0}\left(A_{0}+C-A_{1}\right)
\end{aligned}
$$

Sabendo-se que o segmento $M_{0} B_{1}$ também pode ser representado por K.P. $P_{0}$ como foi descrito na expressão (5), e que $C=M_{0} B_{1}$, tem-se:

$$
Y=0,5 Q_{0}\left(A_{0}+K P_{0}-A_{1}\right)
$$


Dessa forma, a área $Y\left(A_{0} M_{0} B_{1} A_{1} A_{0}\right)$ é representada pela equação abaixo:

$$
Y=0,5 Q_{0}\left(K P_{0}+A_{0}-A_{1}\right)
$$

A área $X$ pode ser representada pela área geométrica de um triângulo (vide Figura 3), tomando-se 0 segmento $M_{0} B_{1}$ como a base do triângulo e a distância $\left(Q_{1}-Q_{0}\right)$ como a altura do triângulo. Dessa forma, chega-se à expressão (7) :

$$
X=0,5 Q_{0} \cdot K P_{0}\left(Q_{1}-Q_{0}\right)
$$

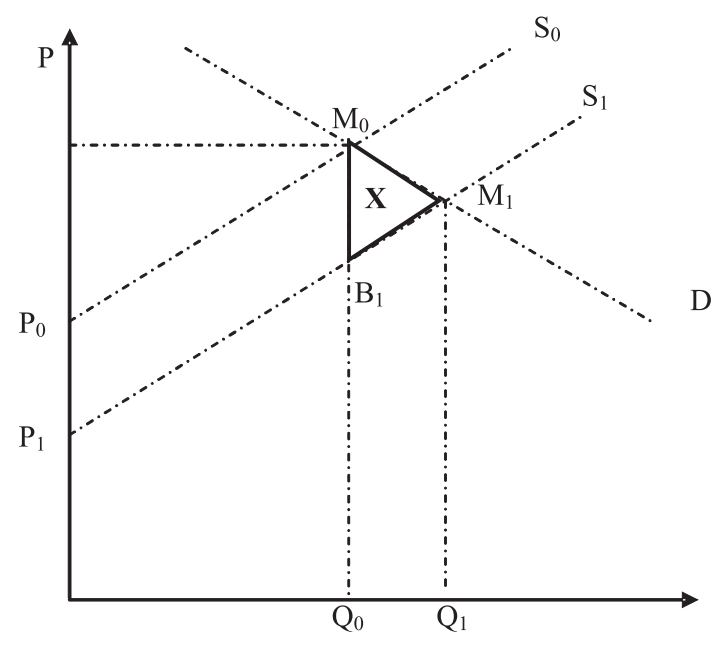

Figura 3 - Demonstração geométrica do cálculo da área X.

Para um deslocamento paralelo da curva de oferta, a mudança da origem influencia a área $Y\left(A_{0} M_{0} B_{1} A_{1} A_{0}\right)$. Seguindo as derivações acima, para uma mudança paralela, $\mathrm{A}_{0} \mathrm{~A}_{1}$ é igual a $\mathrm{KP}_{0}$. Assim,

$$
A_{0}=A_{1}+K P_{0}
$$

Substituindo (6) em (8), tem-se a expressão do benefício social total (BST) para um deslocamento paralelo da curva de oferta, representado pela equação abaixo:

$$
Y=0,5 Q_{0}\left(K P_{0}+A_{1}+K P_{0}-A_{1}\right) \quad \text { ou } \quad Y_{\text {pararelo }}=K P_{0} Q_{0}
$$


Para encontrar o BST para um deslocamento paralelo, soma-se a expressão (7) com a expressão (9), obtendo:

$$
B S T_{\text {pararelo }}: X+Y=0,5 Q_{0} K P_{0}\left(Q_{1}+Q_{0}\right)
$$

Para um deslocamento pivotal da curva de oferta, tem-se que:

$$
A_{1}=A_{0}
$$

Dessa forma, substituindo (11) em (6), tem-se para um deslocamento pivotal da curva de oferta:

$$
\begin{aligned}
& Y=0,5 Q_{0}\left(K P_{0}+A_{0}-A_{0}\right) \\
& Y_{\text {pivotal }}=0,5 K P_{0} Q_{0}
\end{aligned}
$$

Assim, o BST para um deslocamento pivotal da curva de oferta é dado pela soma da expressão (7) com a expressão (12), obtendo:

$$
B S T_{\text {pivotal }}=X+Y_{\text {pivotal }}
$$

Conforme Rose (1980, apud Abdallah, 1998, p.123), o ponto de equilíbrio após a política implementada é obtido da seguinte maneira:

$$
\begin{aligned}
& \left.P_{1}=P_{0}\left[1-\left(K \varepsilon^{s}\right) / \varepsilon^{d}+\varepsilon^{s}\right)\right] \\
& \left.Q_{1}=Q_{0}\left[1+\left(K \varepsilon^{s} \varepsilon^{d}\right) / \varepsilon^{d}+\varepsilon^{s}\right)\right]
\end{aligned}
$$

Substituindo a equação (15) nas equações (10) e (13) e fazendo $Z=(K \varepsilon s) /(\varepsilon d+\varepsilon s)$, obtêm-se as expressões abaixo, representando, respectivamente, o benefício social total para uma mudança paralela e pivotal da curva de oferta:

$$
\begin{aligned}
& B S T_{\text {paralelo }}=0,5 K P_{0} Q_{0}\left(2+Z \varepsilon^{d}\right) \\
& B S T_{\text {pivotal }}=0,5 K P_{0} Q_{0}\left(1+Z \varepsilon^{d}\right)
\end{aligned}
$$

Onde:

$\mathrm{P}_{0}=$ preço de equilíbrio antes da implantação da política analisada;

$\mathrm{Q}_{0}=$ quantidade de equilíbrio antes da implantação da política analisada; 
$\varepsilon^{d}=$ elasticidade-preço da demanda do produto, em valor absoluto:

$\varepsilon^{s}=$ elasticidade-preço da oferta do produto em valor absoluto;

$\mathrm{K}=$ deslocador da curva de oferta e medidor da mudança proporcional do custo unitário;

$Z$ = relação entre o deslocador da curva de oferta e as elasticidades-preço da demanda e da oferta, em valor absoluto;

$\mathrm{BST}=$ benefício social total bruto, em R\$;

A equação (4) mostra que $K$ é medido pela mudança proporcional na produção, dividida pela elasticidade-preço da oferta $\left(\varepsilon^{S}\right)$. Portanto, para calcular $K$, é necessária uma estimativa do produto ofertado ao preço $\mathrm{P}_{0}$ antes e após a implantação da política de crédito rural destinada à avicultura no Brasil.

\section{M etodologia}

\subsection{Fonte de dados}

O estudo foi realizado com base em dados secundários de origem das seguintes fontes: Fundação Getúlio Vargas (FGV), Empresa Brasileira de Pesquisa Agropecuária (Embrapa), Instituto Brasileiro de Geografia e Estatística (IBGE), Banco Central (BACEN), União Brasileira de Avicultura (UBA), Associação Brasileira dos Produtores e Exportadores de Frango (ABEF), Anuário da Avicultura Industrial e outras instituições atuantes do setor.

Além disso, foram realizadas consultas a periódicos especializados e à Internet.

\subsection{Definição das variáveis}

O presente estudo utilizou uma série histórica de dados mensais, considerando o período de janeiro de 1996 a dezembro de 2004, englobando o período de ampliação da política de crédito rural que compreende os meses de janeiro de 1997 a dezembro de 2004.

A definição e a operacionalização das variáveis utilizadas no modelo são descritas a seguir:

- As variáveis quantidade demandada de carne de frango (QDft) e quantidade ofertada de carne de frango ( $Q S f t)$ foram construídas a partir dos dados de produção, importação e exportação de carne de frango, em toneladas, e transformados em $\mathrm{kg}$. Os dados foram obtidos junto à Associação Brasileira dos Produtores e Exportadores 
de Frango e ao Anuário Estatístico de Avicultura dos anos de 2002, 2004 e 2006;

- A variável preço da carne de frango (Pft) foi disponibilizada pela Fundação Getúlio Vargas, em valor monetário mensal corrente, em tonelada, e transformada em valores referentes a reais de agosto de 2006, pelo INPC, por kg;

- A variável preço da carne suína (PSt), que representa o preço do bem substituto, foi disponibilizada pela Fundação Getúlio Vargas em valor monetário mensal corrente, por tonelada, e transformada em valores referentes a reais de agosto de 2006, pelo INPC, por kg;

- A variável renda nacional (RT), que representa os valores mensais do Produto Interno Bruto, a preço de mercado, foi obtida junto ao Banco Central (BACEN) em valor monetário mensal corrente e transformada em valores referentes a reais de agosto de 2006, pelo INPC;

- A variável $(P O P t)^{1}$ representa a população residente do Brasil em número de habitantes. Tal variável foi construída a partir de dados anuais retirados do banco de dados macroeconômicos do Instituto de Pesquisa Econômica Aplicada (IPEADATA) e transformados em dados mensais;

- A variável (Rt) preço da ração representa o custo de produção. Segundo Brum et al. (1993), essa variável é responsável, aproximadamente, por $70 \%$ do custo de produção da carne de frango. Esta variável foi construída a parir de dados mensais do preço da soja, por tonelada obtidos junto à Fundação Getúlio Vargas e transformada em valores referentes a reais de agosto de 2006, pelo IN PC, por kg;

- A variável crédito rural (CRt) foi construída pela soma do crédito rural de custeio e do crédito para investimento destinados à avicultura brasileira. Esses dados foram extraídos do Anuário Estatístico de Crédito Rural fornecido pelo Banco Central (BACEN), em valores anuais correntes e, posteriormente, transformados em valores mensais ${ }^{2}$ em $\mathrm{R} \$$ de agosto de 2006.

\subsection{Especificação do modelo}

$\mathrm{Na}$ determinação das elasticidades-preço da demanda e da oferta de carne de frango foi utilizado o modelo log-linear de equações simultâneas. Para isso empregou-se o método dos mínimos quadrados

\footnotetext{
A variável população foi construída a partir da taxa de variação anual, a qual foi transformada em mensal e aplicada à potência do respectivo mês.

$2 \mathrm{Na}$ transformação dos valores anuais do crédito rural em valores mensais foi utilizada a mesma metodologia da variável população.
} 
de dois estágios (MQ2E), supondo um modelo de equilíbrio em que o preço e a quantidade são determinados ao mesmo tempo, tanto para a demanda quanto para a oferta.

Neste estudo, as funções de demanda e de oferta foram definidas, respectivamente, por:

$$
\begin{aligned}
& \operatorname{Ln}\left(Q^{D} f\right) t=f(\operatorname{Ln}(P f t), \operatorname{Ln}(P S t), \operatorname{Ln}(R T t), \operatorname{Ln}(P O P t)) \\
& \operatorname{Ln}\left(Q^{S} f\right) t=f(\operatorname{Ln}(P f t), \operatorname{Ln}(P R t), \operatorname{Ln}(C R t))
\end{aligned}
$$

Sendo o equilíbrio de mercado é dado por:

$$
\operatorname{Ln}\left(Q^{D} f\right)=\operatorname{Ln}\left(Q^{S} f\right)=\operatorname{Ln}(Q f)
$$

Onde:

$\operatorname{Ln}\left(Q^{D f}\right) t=$ Logaritmo natural da quantidade demandada de carne de frango no mês $\mathrm{t}$, em $\mathrm{kg}$.

$\mathrm{Ln}\left(\mathrm{Q}^{\mathrm{S} f}\right) \mathrm{t}=$ Logaritmo natural da quantidade ofertada de carne de frango no mês $\mathrm{t}$, em $\mathrm{kg}$.

$\operatorname{Ln}(\mathrm{Q} f) t=$ Logaritmo natural da quantidade de equilíbrio de carne de frango no mês $\mathrm{t}$, em $\mathrm{kg}$.

$\mathrm{LnPft}=$ Logaritmo natural do preço do $\mathrm{kg}$ da carne de frango no mês t, em $R \$$.

LnPSt = Logaritmo natural do preço do $\mathrm{kg}$ da carne suína no mês t, em R\$.

LnRTt $=$ Logaritmo natural do produto interno bruto a preço de mercado no mês $t$, em $R \$$.

LnPOPt = Logaritmo natural da população residente no Brasil, no mês $t$, em $n^{\circ}$ de habitantes.

$\mathrm{LnRt}=$ Logaritmo natural do preço da soja por Kg, no mêst, em R\$.

LnCRt = Logaritmo natural da soma do crédito rural de custeio e de investimento, disponibilizados para o setor avícola no Brasil, no mês t, em R\$.

Os valores monetários foram apresentados em $\mathrm{R} \$$ de agosto de 2006, corrigidos pelo INPC.

Como o modelo apresentou duas equações estruturais e duas variáveis endógenas, trata-se de um modelo completo. Contudo, para que fosse possível estimar os parâmetros das equações de demanda e oferta de carne de frango no Brasil, o modelo econômico foi transformado em um modelo estatístico, tal como o descrito a seguir: 
Equação de demanda:

$Q D f t=\alpha_{0}+\alpha_{1} P f t+\alpha_{2} P S t+\alpha_{3} R T t+\alpha_{4} P O P t+u_{1} t$

Equação de oferta:

$$
Q S f t=\beta_{0}+\beta_{1} P f t+\beta_{2} R t+\beta_{3} C R t+u_{2} t
$$

As equações (20) e (21) são denominadas de equações estruturais, os a's e $\beta^{\prime}$ s correspondem aos parâmetros estruturais e $u_{1}$ e $u_{2}$ são os erros estatísticos ou termos de perturbações aleatórias, supostos normalmente distribuídos, com média zero e variância constante, das equações de demanda e oferta, respectivamente.

As variáveis endógenas no modelo corresponderam ao Preço (PFt) e Quantidade ( $\mathrm{Qft}$ ) de carne de frango, sendo exógenas as demais variáveis.

$\mathrm{Na}$ estimação desse modelo econométrico, esperaram-se os seguintes sinais:

Para a Demanda:

$$
\alpha_{0} \geq 0, \alpha_{1}<0, \alpha_{2}>0, \alpha_{3}<0 \text { e } \alpha_{4}>0
$$

Para a oferta:

$$
\beta_{0} \geq 0, \beta_{1}>0, \beta_{2}<0, \beta_{3}>0
$$

Neste sistema de equações simultâneas, o método dos mínimos quadrados não foi mais viável. Como a variável preço da carne de frango (PFt) apresentou correlação com a perturbação, as estimativas dos parâmetros estruturais geradas pelo método dos mínimos quadrados ordinários tornaram-se inconsistentes. Contudo, este problema foi contornado através da utilização do método dos mínimos quadrados de dois estágios, que consiste na aplicação do método dos mínimos quadrados ordinários duas vezes.

Primeiro estimou-se a equação na forma reduzida, na qual a variável endógena (Pft) apareceu como dependente de todas as variáveis exógenas e das perturbações do sistema.

\section{Estágio:}

$$
P f t=c_{0} P S t+c_{2} R T t+c_{3} P O P t+c_{4} R t+c_{5} C R t+S t
$$


Onde:

$$
\begin{aligned}
& c_{0}=\left(\beta_{0}-\alpha_{0}\right) /\left(\alpha_{1}-\beta_{1}\right) \\
& c_{1}=\beta_{2} /\left(\alpha_{1}-\beta_{1}\right) \\
& c_{2}=-\alpha_{2} /\left(\alpha_{1}-\beta_{1}\right) \\
& c_{3}=-\alpha_{3} /\left(\alpha_{1}-\beta_{1}\right) \\
& c_{4}=-\alpha_{4} /\left(\alpha_{1}-\beta_{1}\right) \\
& c_{5}=\beta_{3} /\left(\alpha_{1}-\beta_{1}\right) \\
& S t=\left(u_{1}-u_{2}\right) /\left(\alpha_{1}-\beta_{1}\right)
\end{aligned}
$$

Os $\mathrm{Ci}_{\mathrm{s}}$ representaram os coeficientes da equação na forma reduzida e $S_{t}$ a perturbação da referida equação.

A partir dos valores desses parâmetros estimados e nos valores de todas as variáveis predeterminadas foram obtidos os valores estimados Pt.

No segundo estágio, os valores observados da variável endógena (Pt) foram substituídos por Pt. Os coeficientes das equações estruturais foram, então, estimados pelo método dos mínimos quadrados ordinários, usando os valores estimados da variável endógena Pt e os valores observados das variáveis predeterminadas.

Nesta pesquisa, as equações do segundo estágio foram obtidas da seguinte forma.

\section{2을ágio:}

Equação de Demanda:

$$
Q D f t=\alpha_{0}+\alpha_{1} \hat{P} f t+\alpha_{2} P S t+\alpha_{3} R T t+\alpha_{4} P O P t+u_{1} t
$$

Equação de Oferta:

$$
Q S f t=\beta_{0}+\beta_{1} \hat{P} f t+\beta_{2} R t+\beta_{3} C R t+u_{2} t
$$

Os testes estatísticos aplicados às equações do modelo de equações simultâneas desta pesquisa foram utilizados apenas como indicadores. Segundo Pyndick e Rubinfield (2004), uma vez que os testes habituais não são estritamente válidos em modelos simultâneos como nos modelos uniequacionais. 


\subsection{Cálculo do benefício social total}

Para calcular o beneficio social total (BST) da ampliação de crédito rural destinado à avicultura brasileira, no período de janeiro de 1997 a dezembro de 2004, foram usadas as seguintes equações:

$$
\begin{array}{ll}
B S T=0,5 K P_{0} Q_{0}\left(2+Z \varepsilon^{d}\right) & \begin{array}{l}
\text { para o deslocamento paralelo da } \\
\text { curva de oferta de carne de frango }
\end{array} \\
B S T=0,5 K P_{0} Q_{0}\left(1+Z \varepsilon^{d}\right) & \begin{array}{l}
\text { para o deslocamento pivotal da } \\
\text { curva de oferta de carne de frango }
\end{array}
\end{array}
$$

Onde:

$\mathrm{P}_{0}=$ preço de equilíbrio antes da implantação da política de ampliação do crédito rural;

$\mathrm{Q}_{0}=$ quantidade de equilíbrio antes da implantação da política de ampliação do crédito rural;

$\varepsilon^{d}=$ elasticidade-preço da demanda do produto analisado, em valor absoluto;

$\varepsilon^{s}=$ elasticidade-preço da oferta do produto analisado;

$\mathrm{K}=$ deslocador da curva de oferta e medidor da mudança proporcional do custo unitário;

$Z=$ relação entre o deslocador da curva de oferta e as elasticidades-preço da demanda e da oferta, em valor absoluto;

$\mathrm{BST}=$ benefício social total bruto em $\mathrm{R} \$$.

O valor de $\mathrm{K}$ foi calculado para o período de janeiro de 1997 a dezembro de 2004. Considerando o mês de dezembro de 1996 como o período zero (pois é o primeiro mês anterior à política de ampliação do crédito rural destinado à avicultura), encontrou-se a seguinte equação para calcular Q²t para o período janeiro de 1997 a dezembro de 2004:

$$
\operatorname{Ln} Q^{s} 2 t_{(d e z / 2004)}=\beta_{1}+\beta_{2} \operatorname{LnPf} f_{(d e z / 1996)}+\beta_{3} \operatorname{Ln} R_{(d e z / 2004)}+\beta_{4} \operatorname{LnC} r_{(d e z / 2004)}
$$

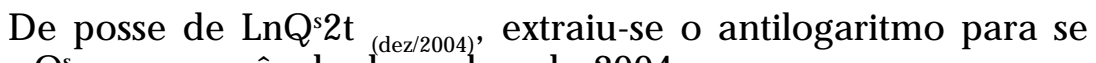
obter $\mathrm{Q}_{2 \mathrm{t}}^{\mathrm{s}}$ para o mês de dezembro de 2004.

Com 0 valor de $\mathrm{Q}_{2 \text { (dezl 2004), }}^{\mathrm{s}}$ pôde-se calcular $\mathrm{k}$ o período de janeiro de 1997 a dezembro de 2004 da seguinte forma:

$$
K=\frac{\left(1-\frac{Q_{0(d e z / 1996)}}{Q_{2(d e z / 2004)}^{s}}\right)}{\varepsilon^{s}}
$$


Substituindo-se o valor de K na expressão abaixo, pôde-se calcular Z, como segue:

$$
Z=\frac{K \cdot \varepsilon^{s}}{\left(\varepsilon^{s}+\varepsilon^{d}\right)}
$$

Com os valores de $K Z, \varepsilon^{d}, P_{0}$ e $Q_{0}$ (considerando o mês de dezl 1996 como sendo o períódo zero), calculou-se o benefício total para o período de janeiro de 1997 a dezembro de 2004, como segue abaixo:

Admitindo um deslocamento paralelo:

$$
B S T_{(d e z / 2004)}=0,5 K_{(d e z / 2004)} P_{0(d e z / 1996)} Q_{0(d e z / 1996)}\left(2+Z_{(d e z / 2004)} \varepsilon^{d}\right)
$$

Admitindo um deslocamento pivotal:

$$
B S T_{(d e z / 2004)}=0,5 K_{(d e z / 2004)} P_{0(d e z / 1996)} Q_{0(d e z / 1996)}\left(1+Z_{(d e z / 2004)} \varepsilon^{d}\right)
$$

\section{Resultados e Discussão}

\subsection{A nálise da equação estimada de demanda de carne de frango para 0} Brasil

O poder explicativo da regressão, dado pelo coeficiente de determinação ajustado, foi de 0,857 , indicando um bom ajustamento da função demanda, ou seja, que $85,7 \%$ das variações na quantidade demandada de carne de frango são explicadas pela variação conjunta das variáveis Pft, PSt, RTt e POPt.

Os resultados obtidos na equação estrutural de demanda de carne de frango no Brasil, após correção de autocorrelação serial nos erros e heterocedasticidade, ${ }^{3}$ encontram-se na Tabela 1 , na qual observa-se que os sinais dos coeficientes estão de acordo com a teoria econômica. A identificação da autocorrelação foi feita através do teste Durbin tendo sido considerada correlação de primeira ordem. Os coeficientes das variáveis explicativas apresentaram valores superiores aos seus respectivos erros-padrão.

\footnotetext{
3 Estes problemas foram analisados através teste White para heterocedasticidade, Durbin Watson para autocorrelação serial e matriz de correlação para multicolinearidade.
} 
A elasticidade-preço da demanda apresentou coeficiente associado à variável preço real da carne de frango de $-1,117$, o que indica que se houver um aumento de $10 \%$ no preço real da carne de frango a quantidade demandada da carne de frango sofrerá uma redução de $11,2 \%$, ceteris paribus. Dessa forma, conclui-se que a demanda brasileira por carne de frango no período analisado foi elástica.

Os resultados obtidos por Castro (1991), que estimou a oferta e demanda de frango no Brasil, e Triches et. al. (2004), que estimaram a função de demanda de carne de frango da Serra Gaúcha (RS), indicaram que a demanda por carne de frango é elástica.

Existem algumas características que tornam a demanda por carne de frango elástica, podendo-se destacar a disponibilidade de um grande número de substitutos, tais como: carne suína, carne bovina, ovos, peixes e o período de tempo em que está sendo analisada a demanda por determinado produto, períodos mais longos tendem a gerar demandas elásticas, pois o consumidor pode ter um maior conhecimento à respeito das alternativas de consumo existentes. Nesta pesquisa, foi utilizada uma série mensal de janeiro de 1996 a dezembro de 2004, ou seja, uma série de 108 meses, o que favorece a obtenção de elasticidades maiores que 1 .

Como pode-se verificar, conforme dados da Associação Brasileira de Produtores e Exportadores de Frango - ABEF - (2005), o consumo interno de frango aumentou consideravelmente nos últimos 16 anos, principalmente pelos ganhos de produtividade alcançados pela indústria de alimentos que provocou forte redução no preço do produto, variável determinante neste processo, já que frango se tornou mais barato em relação às outras carnes, tornando uma alternativa acessível de consumo protéico.

Com relação à elasticidade-preço-cruzada da demanda, coeficiente associado a variável preço real da carne de suína foi de 0,226, o que indica que se houver um acréscimo de $10 \%$ no preço real da carne suína a quantidade demandada da carne de frango sofrerá um aumento de $2,3 \%$, ceteris paribus. Portanto conclui-se que as carnes de frango e suína são bens substitutos entre si. Resultado similar foi encontrado por Triches et. al. (2004). 
TABELA 1 - Equação estimada de demanda de carne de frango no Brasil, de janeiro de 1996 a dezembro de 2004.

\begin{tabular}{|c|c|c|c|c|}
\hline \multicolumn{5}{|c|}{ V ariável Dependente: $\operatorname{Ln}(\mathrm{Q} \mathrm{dft})$} \\
\hline Variável & $\begin{array}{l}\text { Coeficiente } \\
\text { estimado }\end{array}$ & Erro padrão & Estatística T & Prob. \\
\hline Constante $\boldsymbol{\alpha}_{0}$ & $-101,3004$ & 13,27908 & $-7,628570$ & 0.0000 \\
\hline$\hat{\operatorname{Ln}}(\mathrm{Pft})$ & $-1,117710$ & 0,423316 & $-2,640368$ & 0.0096 \\
\hline $\operatorname{Ln}(\mathrm{PSt})$ & 0,226768 & 0,165668 & 1,368811 & 0.1741 \\
\hline $\operatorname{Ln}(R T t)$ & 0,193649 & 0,143776 & 1,346882 & 0.1810 \\
\hline $\operatorname{Ln}(\mathrm{PO} \mathrm{Pt})$ & 6,150927 & 0,776093 & 7,925501 & 0.0000 \\
\hline$R^{2}$ & 0,863998 & & & \\
\hline$\overline{\mathrm{R}^{2}}$ & 0,857265 & \multicolumn{2}{|c|}{ Estatística F } & 128,3271 \\
\hline Durbin-W atson & 2,013567 & \multicolumn{2}{|c|}{ Prob(Estatística F) } & 0,000000 \\
\hline
\end{tabular}

Fonte: Resultados da pesquisa.

Em relação à elasticidade-renda da demanda, o coeficiente associado à variável renda real agregada da população brasileira foi igual a 0,193 que indica que se houver um incremento de $10 \%$ na renda real agregada da população do país a quantidade demandada da carne de frango sofrerá uma elevação de 1,9\%, ceteris paribus. Sendo assim, classifica-se a carne de frango como um bem normal. Este resultado encontra-se em conformidade com os obtidos por Triches et. al. (2004).

O resultado exposto acima se traduz na atual mudança de hábitos alimentares, pois os consumidores estão cada vez mais exigentes, preferindo alimentos que contenham proteínas nobres, de fácil digestão, carnes com baixo teor de gorduras, fato esse que fez com que a carne de frango deixasse de ser a principal fonte de alimento das camadas mais pobres da população, quando então era considerada um bem inferior, passando a compor o cardápio das classes mais privilegiadas.

O coeficiente associado à variável população residente do país equivalente a 6,150 sugere que se houver uma expansão de $10 \%$ na população residente do país a quantidade demandada da carne de frango sofrerá uma ampliação de $61,5 \%$, ceteris paribus. 


\subsection{A nálise da equação estimada de oferta de carne de frango para o Brasil}

As variáveis explicativas utilizadas para estimar a equação de oferta por carne de frango ( $\mathrm{Q}$ stt) foram o preço real estimado da carne de frango (Pft), o preço real da ração (Rt) e o crédito rural destinado à avicultura (CRt). 0 poder explicativo da regressão, dado pelo coeficiente de determinação ajustado, foi de 0,832 , indicando um bom ajustamento da função oferta, ou seja, que $83,2 \%$ das variações na quantidade ofertada de carne de frango são explicados pela variação conjunta nas variáveis Pft, PRt e CRt.

Os testes de violação dos pressupostos demonstraram que o modelo possui variância residual constante, ausência de multicolinearidade e inexistência de autocorrelação serial entre os resíduos.

Os resultados obtidos na equação estrutural de oferta de carne de frango no Brasil encontram-se na Tabela 2, na qual se observa que os sinais dos coeficientes das variáveis preço real estimado da carne de frango ( $\mathrm{Pft}$ e crédito rural destinado à avicultura (CRt), estão de acordo com a teoria econômica. Este resultado não ocorreu com o coeficiente da variável preço real da ração, pois indicou uma relação direta entre a variável preço real da ração e da quantidade produzida de carne de frango. No entanto, do ponto de vista estatístico, este coeficiente não se mostrou significante. Acredita-se que isso tenha ocorrido em decorrência de a taxa de crescimento do preço da carne de frango ser maior que a de crescimento do preço da ração, no período de estudo.

TABELA 2 - Equação estimada de oferta de carne de frango no Brasil, de janeiro de 1996 a dezembro de 2004.

\begin{tabular}{|c|c|c|c|c|}
\hline \multicolumn{5}{|c|}{ V ariável Dependente: $\operatorname{Ln}(\mathrm{Q} S \mathrm{ft})$} \\
\hline Variável & Coeficiente & Erro padrão & Estatística T & Prob. \\
\hline$\beta_{0}$ & 14,04713 & 1,526587 & 9,201655 & 0,0000 \\
\hline$\widehat{\operatorname{Ln}(P f t)}$ & 1,298292 & 0,320751 & 4,047669 & 0,0001 \\
\hline $\operatorname{Ln}(R t)$ & 0,005281 & 0,081819 & 0,064551 & 0,9487 \\
\hline $\operatorname{Ln}(C R t)$ & 0,253331 & 0,079416 & 3,189916 & 0,0019 \\
\hline $\mathrm{R}^{2}$ & 0,832538 & & & \\
\hline$\overline{\mathrm{R}^{2}}$ & 0,825971 & \multicolumn{2}{|c|}{ Estatística F } & 126,7732 \\
\hline Durbin-W atson & 2,200970 & \multicolumn{2}{|c|}{ Prob (Estatística F) } & 0,000000 \\
\hline
\end{tabular}

Fonte: Resultados da pesquisa. 
Quanto à elasticidade-preço da oferta, o coeficiente associado à variável preço real da carne de frango de 1,298 revela que, se houver um aumento de $10 \%$ no preço real da carne de frango, a quantidade ofertada sofrerá ceteris paribus, um incremento de $13 \%$. Dessa forma, conclui-se que a oferta de carne de frango é elástica.

Comparando o resultado exposto com o coeficiente de elasticidade-preço da oferta de carne de frango do Brasil obtido por Castro (1991) de 0,74, sugerindo que a oferta a se apresentou inelástica, percebe-se que houve divergência do resultado obtido nesta pesquisa. Tal divergência pode ter ocorrido em virtude do aumento, a partir dos anos 1990, do número de empresas produtoras e/ou beneficiadoras de carne de frango no Brasil.

O coeficiente, associado à variável crédito rural destinado à avicultura equivalente à 0,253 , indica que se houver uma expansão de $10 \%$ no crédito rural destinado à avicultura, a oferta de carne aumentará 2,5\%, ceteris paribus.

No período pós-política de ampliação do crédito rural, período compreendido entre janeiro de 1997 e dezembro de 2004, verificou-se que o preço da carne de frango sofreu um aumento de $29 \%$, enquanto a quantidade ofertada de carne frango ampliou em $98 \%$. Contudo, a elasticidade-preço da oferta estimada nesta pesquisa foi de 1,29 . Um aumento de $29 \%$ no preço da carne de frango ocasionaria uma elevação de $37,41 \%$ na quantidade ofertada desse produto, ceteris paribus.

Este fato sugere que existem outras variáveis, com exceção do preço, que estão influenciando a expansão da quantidade ofertada de carne de frango no Brasil. 0 crédito rural está entre essas variáveis que influenciaram tal expansão.

\subsection{A nálise do benefício social total}

\subsubsection{M ensuração do benefício social total}

A partir da função de oferta estimada na Tabela 2, calculou-se a quantidade ofertada em nível $\mathrm{P}_{0}$ para 0 mês de dezembro de 2004, dada por $Q_{2}^{s}$ (dez/2004), pela seguinte equação:

$$
\operatorname{Ln} \hat{Q}_{2(d e z / 2004)}^{s}=13,87+1,30 \operatorname{LnPft}_{(d e z / 1996)}+0,01 \operatorname{LnR} t_{(d e z / 2004)}+0,25 \operatorname{LnCRt_{(dez/2004)}}
$$

Extraído o antilog de LnQ2S(dez/2004) pôde-se calcular o valor escalar de K. De posse de K, do preço observado em dez/1996 [Pfto(dez/ 
1996)], da quantidade observada em dez/1996 [Q0(dez/1996)] e das elasticidades-preço da demanda e da oferta, $\varepsilon^{d}$ e $\varepsilon^{s}$, respectivamente, foram obtidos os valores de $P_{1}$ e $Q_{1}$ através das equações (14) e (15).

A Tabela 3 fornece os valores de $Q_{2}{ }^{5}\left(\right.$ dez/2004), $K, P_{1}, Q_{1}$ e BST para os deslocamentos paralelo e pivotal da curva de oferta de carne de frango, a partir da política de crédito rural destinada à avicultura brasileira.

O benefício social gerado pela política de ampliação do crédito rural destinado à avicultura, considerado a partir de janeiro de 1997 até dezembro de 2004 , totalizou $\mathrm{R} \$ 186.926 .769,54$ para um deslocamento paralelo da curva de oferta de carne de frango.

TABELA 3 - Benefício social total estimado para o deslocamento paralelo e pivotal da curva de oferta de carne de frango no Brasil, de janeiro de 1997 a dezembro de 2004.

\begin{tabular}{c|c|c|c|c|c}
\hline \multicolumn{2}{c}{} & \multicolumn{2}{c}{ BEN EFICI0 SO CIA L TO TA L } \\
\cline { 4 - 6 } \multicolumn{2}{c|}{} & Deslocamento Paralelo & Deslocamento Pivotal \\
\hline$Q_{2}{ }^{5}$ (dez/2004) & $K$ & $P 1$ & $Q 1$ & BST & BST \\
\hline (Kg) & & $(\mathrm{R} \$)$ & $\mathrm{Kg}$ & $(\mathrm{R} \$)$ & $(\mathrm{R} \$)$ \\
\hline $566.944 .803,15$ & 0,3 & 1,4 & $361.927 .288,96$ & $186.926 .769,54$ & $102.135 .880,94$ \\
\hline
\end{tabular}

Fonte: Resultados da Pesquisa

Considerando um deslocamento pivotal da curva de oferta, o benefício social foi de $\mathrm{R} \$ 102.135 .880,94$. O deslocamento pivotal da curva de oferta de carne de frango implica que os custos médios se mantiveram constantes após o incentivo.

O benefício gerado a partir da expansão da oferta, considerando a redução nos custos médios de produção, supera em $83 \%$ o benefício gerado a partir da expansão da curva de oferta, considerando constante o custo médio de produção.

\section{Considerações Finais}

A expansão do crédito rural destinado à avicultura Brasileira ampliou a oferta de frango no Brasil no período de janeiro de 1997 a dezembro de 2004 e gerou bem-estar social através do aumento do excedente tanto do consumidor como do produtor, o que sugere a viabilidade desta atividade como fator de desenvolvimento econômico do meio rural brasileiro. 
É relevante ressaltar, no entanto, que o estudo apresentou como fator limitante a operacionalização dos dados utilizados. Empregou-se séries resultantes de transformações de dados anuais de quantidades em dados mensais. Em trabalhos futuros, sugere-se o uso de uma série maior com dados originalmente anuais.

\section{Referências}

ABDALLAH, P. R. Atividade Pesqueira no Brasil: Política e Evolução. Piracicaba, São Paulo, 1998. Tese de doutorado apresentada à Escola Superior Luís de Queiroz, Universidade de São Paulo.

AKINO, M \& HAYAMI, Y. Eficiency and Equity in Public Research: Rice Breeding in J apan's Economic Development. American J ournal of Agricultural Economics. Vol. 57, No. 1, p. 1-10, fev. de 1975.

ABEF - Associação Brasileira dos Produtores e Exportadores de frango. Relatórios anuais 2000 - 2005. Disponível em: http://www.abef.com.br/Relatorios_Anuais.asp. < acessado em <20/08/2006>

ANUÁRIO 1998 DA AVICULTURA INDUSTRIAL. Porto Feliz: Gessulli Agribusiness, 1997.

ANUÁRIO 2002 DA AVICULTURA INDUSTRIAL. Porto Feliz: Gessulli Agribusiness, 2002.

ANUÁRIO 2004 DA AVICULTURA INDUSTRIAL. Porto Feliz: Gessulli Agribusiness, 2004.

ANUÁRIO 2005 DA AVICULTURA INDUSTRIAL. Porto Feliz: Gessulli Agribusiness, 2005.

ANUÁRIO ESTATÍSTICO DE CRÉDITO RURAL 1996 - 1998, BANCO CENTRAL DO BRASIL, Brasilia.

ANUÁRIO ESTATÍSTICO DE CRÉDITO RURAL 1999 - 2004, BANCO CENTRAL DO BRASIL, Brasília. Disponível em: http://www.bcb.gov.br/?RED 1-RELRURAL < acesso em $<07 / 08 / 2006>$

AVIMIG - Associação dos Avicultores de Minas Gerais. Proposta para Presidente da República eleito em 29/10/06 Avicultura brasileira. Disponível em

$<$ Acessado em 20/03/2007>

AYER \& SCHUH, C. E. Social rates of return and other aspects of agricultural research: The case of cotton research in São Paulo, Brasil. American J ournal of Agricultural Economics. 54(4): 557-569, 1972.

BRUM, P. et al.. Uso do farelo de arroz integral em dietas para frango de corte. Comunicado Técnico 201. Centro Nacional de Pesquisa de Suínos e Aves. Pág. 1-2, 1993.

CASTRO, N. G. O ferta e Demanda de Frango de Corte no Brasil. Lavras, Minas Gerais, 1991. Dissertação de Mestrado apresentada à Escola Superior de Lavras.

CRUZ, E. R. da, et al.. Taxas de retorno dos investimentos da EMBRAPA: Investimentos totais e capital físico. Brasília, 1982.

CRUZ, E. R. da; ÁVILA, AFD. Retornos dos investimentos em pesquisa agropecuária na área de abrangência do Projeto I EM BRAPA/BIRD. Brasília, 1985.

FGV - Fundação Getúlio Vargas. Preços Agropecuários. FGVDADOS. Disponível em http://fgvdados.fgv.br/index.htm. <A cessado em <13/07/2006> 
GRILICHES, Z. Research cost and social returns: hybrid corn and related inovations. J ournal of Política Economy. 66(10) : 419-31, 1958.

HAYAMI, Y.; HERDT, R. Market Price Effects of Technological Change on Income Distribution in Semisubsistence A griculture. American J ournal of Agricultural Economics. Vol. 59, No. 2 , p. 245-256, maio de 1977.

HERTFORD, R.; SCHMITZ, A. Measuring economic returns of agricultural research. Recourse allocation and productivity in national and international agricultural research. Minneapolis, University of Minnesota Press, 1977.

IBGE - Instituto Brasileiro de Geografia e Estatística. Produção Agrícola Municipal 2000. Banco de dados A gregados. Disponível em : www.sidra.ibge.gov.br>. Acesso em 20 de jan. de 2006.

IPEA - Instituto de Pesquisa Econômica Aplicada Dados Macroeconômicos: População residente . Diponível em : http://www.ipeadata.gov.br/ipeaweb.dll/ipeadata?167405140. $<$ Acessado em 20/07/2006>

KHAN, A. S.; SOUZA, J. S. Taxa de retorno social do investimento em pesquisa na cultora da mandioca no Nordeste. Revista de Economia e Sociologia Rural. Brasília, 29(4): 411-426,1991.

LINDNER R. K.; J ARRETT, F. G. Supply Shifts and the Size of Research Benefits. American J ournal of Agricultural Economics. V. 60, n.1 p.48-58, fev. de 1978.

Ministério do Planejamento e Orçamento. Mensagem ao congresso Nacional 1998. Disponível em: https://www.presidencia.gov.br/publi_04/CO LECAO/98MENS8.HTM. Acessado em < 15/08/2006>

PINDYCK, R. S.; RUBINFIELD, D. L. Microeconomia. $5^{\circ}$ ed. São Paulo: Makron Books, 2002.

PETERSON, W L. Return to poltry research in the United States. J ournal of Farms Economics. 49(8): 656-661, 1967.

ROSE, R. N. Supply Shifts and research benefits: comment. Amercan J ournal of Agricultural Economics. Vol. 62, No. 4 p. 834-837, nov. de 1980.

SALES, F. M. G. A inadimplência do crédito rural nas áreas de concentração de fruteiras no Estado do Ceará. Monografia do curso de Agronomia apresentada à Universidade Federal do Ceará. Fortaleza. 2005.

SANTANA, A. C.; KHAN, A. S. Avaliação e distribuição dos retornos sociais da adoção tecnológica na cultura de feijão Cauípi no Nordeste. Revista de Economia Rural. Braślia, 25(2): 191-203,1987.

SCHMITZ, A; SECKLER, D. Mecanized Agriculture and Social Welfare: The Case of the Tomato Harvester. American J ournal of Agricultural Economics. Vol. 52, N o. 4, p. 569577, nov de 1970.

SILVA A. S.; KHAN A. S. Benefícios sociais da substituição de milho por raspa de mandioca em ração suína no Estado do Ceará. Revista de Economia e Sociologia Rural, v. 32, no 1 , p. 87-98, 1994.

VARIAN, H. R. Microeconomia: Princípios Básicos. $6^{\circ}$ ed. Rio de J aneiro: Campus, 2003. TRICHES, D. et. al. A cadeia produtiva da Serra Gaúcha: uma análise da estrutura de produção e mercado. Disponível em: http://hermes.ucs.br/ccea/ipes/td/IPES\%20TD\% 20011\%20SET\%202004.pdf. <Acessado em 18/12/2006>

UBA - União Brasileira de Avicultura.Relatório Anual 2004-2005/2006. Disponível em http://www.uba.org.br/. Acessado em <20/08/2006>

Recebido em 20/06/2008.

Aceito em 23/07/2008. 